\title{
Comparative study of overlapping genes in bacteria, with special reference to Rickettsia prowazekii and Rickettsia conorii
}

\author{
Kishore R. Sakharkar, ${ }^{1}$ Meena K. Sakharkar, ${ }^{2}$ Chandra Verma $^{1}$ \\ and Vincent T. K. Chow ${ }^{3}$ \\ ${ }^{1}$ Biolnformatics Institute, Matrix, 30 Biopolis Street, Singapore \\ ${ }^{2}$ Nanyang Centre for Supercomputing and Visualization, School of Mechanical and Production \\ Engineering, Nanyang Technological University, Singapore \\ ${ }^{3}$ Human Genome Laboratory, Department of Microbiology, Faculty of Medicine, National \\ University of Singapore, Kent Ridge, Singapore 117597
}

\begin{abstract}
Overlapping genes have been proposed as a means of achieving genome reduction by compressing the maximum amount of information in limited sequence space. In this report, comparative analyses of the overlapping genes of genomes of nine bacteria with different lifestyles were performed. The results clearly suggest that overlapping genes may be a result of evolutionary pressure to minimize genome size. The genomes of two closely related obligatory intracellular parasites - Rickettsia prowazekii and Rickettsia conorii - were investigated further. Detailed analyses of these two genomes revealed that mutations at the ends of coding regions and elimination of intergenic DNA are the main forces that determine overlapping of genes.
\end{abstract}

\section{INTRODUCTION}

Overlapping genes are a common occurrence in prokaryotic genomes (Normark et al., 1983). In overlapping genes, the same DNA sequence encodes two proteins using different reading frames. The evolution of overlapping genes has been approached theoretically and empirically (Sander \& Schulz, 1979; Smith \& Waterman, 1981; Pavesi et al., 1997). Clark et al. (2001) suggested that overlapping genes occur as a result of mutational bias towards deletion. Overlapping genes are more conserved between species than nonoverlapping genes (Lipman, 1997; Yelin et al., 2003), mostly because a mutation in the overlapping region causes changes in both genes, and selection against such mutations should therefore be stronger. Miyata \& Yasunaga (1978) reported that rates of evolution are slower in overlapping genes. Keese \& Gibbs (1992) suspected that overlapping genes arise as a result of overprinting - a process of generating new genes from pre-existing nucleotide sequences (when more than one out-of-phase reading frame may lie in a single nucleotide sequence). The role of the genetic code in generating an open reading frame

Published online ahead of print on 7 January 2005 as DOI 10.1099/ ijs.0.63446-0.

The overlapping gene pairs of the rickettsial species have been classified into four categories. The gene identification number, the gene name, the direction of overlap and the number of overlapping nucleotides have been tabulated for each category and are available as supplementary material in IJSEM Online.
(ORF) inside a coding region has been elaborated (Cebrat et al., 1997). Overlap is thought to be important as (1) a means of compressing the maximum amount of information into short sequences of structural genes and may be a result of evolutionary pressure to minimize genome size and increase the density of genetic information; and (2) as a mechanism for regulating gene expression through translational coupling of functionally related polypeptides (Normark et al., 1983; Chen et al., 1990; Inokuchi et al., 2000). Some ORFs belong to clusters of more than two overlapping ORFs (Normark et al., 1983; Krakauer, 2000). Overlapping genes may evolve as a result of the extension of an ORF caused by a switch to an upstream initiation codon, substitutions in initiation or termination codons, and deletions and frameshifts that eliminate initiation or termination codons (Rogozin et al., 2002). Comparative analysis of the genomes of Mycoplasma genitalium and Mycoplasma pneumoniae showed that most overlapping genes are generated by mutations at the end of coding regions (Fukuda et al., 1999). The analysis revealed that the loss of a stop codon causes the gene to elongate to the next stop codon. Recently, it was suggested that evolution of overlapping genes occurs at a universal mutation rate across bacterial genomes (Fukuda et al., 2003). However, little is known about the origin, evolution and crossspecies conservation of overlapping genes, and about the frequency and genome-wide distribution of overlapping genes in different genomes. We therefore sought to more closely examine how the relative usage of the genome 
changes with genome size in micro-organisms with similar lifestyles. Here, we report the number of overlapping genes in genomes of bacteria with different lifestyles, and compare overlapping genes in two completely sequenced genomes of obligatory intracellular parasites for humans - Rickettsia prowazekii and Rickettsia conorii. This analysis should help explain the role of overlapping genes in obligatory intracellular parasites of humans, and shed light on their evolution and dynamics in bacterial genomes in relation to genome reduction. Analysis of overlapping genes is hampered by sequencing and annotation errors present in genomes, and by the limitations of gene-finding algorithms to handle multiple reading frames (Burge \& Karlin, 1998). The authors thus advise caution in drawing inferences from these data.

\section{METHODS}

The genome sequences of five obligatory intracellular parasites of humans [Chlamydia pneumoniae (Kalman et al., 1999), Chlamydia trachomatis (Stephens et al., 1998), Mycobacterium leprae (Cole et al., 2001), R. prowazekii (Andersson et al., 1998) and R. conorii (Ogata et al., 2001)], two minimal genomes [Mycoplasma genitalium (Fraser et al., 1995) and Mycoplasma pneumoniae (Himmelreich et al., 1996)], one endosymbiotic bacterium (Buchnera aphidicola; Shigenobu et al., 2000) and one free-living bacterium (Clostridium perfringens; Shimizu et al., 2002) were downloaded from the National Center for Biotechnology Information website (ftp://ftp.ncbi.nlm.nih.gov/ genomes/bacteria). The coding sequence (CDS) annotation feature was used for extracting the genes showing overlap. We define overlapping genes as pairs of adjacent genes whose coding regions partly or completely overlap (Table 1). We calculated the percentage of genes that are present as overlapping gene pairs in each genome, and ascertained the direction of their overlap. We selected the two rickettsial species for further detailed analysis on the possible mechanisms for the genes to overlap. We classified the overlapping gene pairs of the rickettsial species into four categories: i.e. (1) gene pairs that occur as overlapping genes in both genomes; (2) gene pairs that overlap in both genomes with different numbers of overlapping bases; (3) gene pairs that overlap in $R$. prowazekii but are split in $R$. conorii; and (4) gene pairs that overlap in $R$. conorii but are split in $R$. prowazekii. The gene identification number, the gene name, the direction of overlap and the number of overlapping nucleotides are tabulated for each category (Supplementary Tables A, B, C, D and E, available in IJSEM Online). The gene pairs that are homologous in one rickettsial species but do not exactly overlap or occur as split genes in another species (either next to each other or at some distance) were manually examined in detail to identify the possible reasons of their emergence during the course of evolution. As there is a high possibility that incorrectly annotated ORFs may be included in the genomic data, we removed 'nongenuine ORFs' from our detailed analysis. We define 'non-genuine ORFs' as those that are annotated as 'hypothetical' or 'unknown' in the genome databases.

\section{RESULTS AND DISCUSSION}

\section{Genomes and overlapping genes}

Reductive evolution is documented in obligatory intracellular parasites as genes become inactivated once their functions are no longer required in highly specialized niches (Andersson \& Andersson, 1999). This process may have naturally defined the minimal gene set for a pathogenic organism based on lifestyle. Very small genomes have adopted obligate associations with the host.

In order to further investigate the retention of overlapping genes in organisms with different and similar lifestyles, we determined the proportion of genomes represented by overlapping genes in five obligatory intracellular parasites, two reduced genomes, one endosymbiont and one freeliving bacterium (Table 1). It was noteworthy that a substantial portion of the genomes is represented by overlapping genes in all the organisms, clearly suggesting an important role for overlapping gene pairs in bacterial genomes. Interestingly, Mycoplasma genitalium has the smallest genome but the largest percentage representation of overlapping gene pairs (Fig. 1). Mycoplasma pneumoniae, the closest relative of Mycoplasma genitalium, also shows a greater proportion of overlapping gene pairs.

Table 1. Number of overlapping genes in the bacterial genomes and their orientation

olp, Overlapping. Direction of overlaps:,$+ \rightarrow$; $-\leftarrow$.

\begin{tabular}{|c|c|c|c|c|c|c|c|c|c|c|}
\hline \multirow[t]{2}{*}{ Bacterial species } & \multirow[t]{2}{*}{$\begin{array}{l}\text { Genome } \\
\text { size }(\mathrm{Mb})\end{array}$} & \multirow[t]{2}{*}{$\begin{array}{l}\text { Total no. } \\
\text { of ORFs }\end{array}$} & \multirow[t]{2}{*}{$\begin{array}{l}\text { No. of olp } \\
\text { gene pairs }\end{array}$} & \multirow[t]{2}{*}{$\begin{array}{l}\text { No. of 'genuine' } \\
\text { olp gene pairs }\end{array}$} & \multicolumn{3}{|c|}{$\begin{array}{c}\text { Orientation of } \\
\text { overlaps (all genes) }\end{array}$} & \multicolumn{3}{|c|}{$\begin{array}{l}\text { Orientation of overlaps } \\
\text { ('genuine' genes) }\end{array}$} \\
\hline & & & & & $++1--$ & +- & -+ & $++1--$ & +- & -+ \\
\hline Rickettsia prowazekii & $1 \cdot 11$ & 901 & 111 & 40 & $61 / 44$ & 3 & 3 & $26 / 14$ & 0 & 0 \\
\hline Rickettsia conorii & $1 \cdot 27$ & 1407 & 225 & 66 & $87 / 110$ & 17 & 11 & $35 / 29$ & 1 & 1 \\
\hline Chlamydia trachomatis & $1 \cdot 018$ & 975 & 173 & 101 & $68 / 68$ & 34 & 3 & $42 / 41$ & 18 & 0 \\
\hline Chlamydia pneumoniae & $1 \cdot 201$ & 1092 & 199 & 107 & $72 / 78$ & 44 & 5 & $36 / 47$ & 21 & 3 \\
\hline Mycobacterium leprae & $3 \cdot 191$ & 2765 & 341 & 179 & $169 / 122$ & 39 & 11 & $95 / 65$ & 16 & 3 \\
\hline Mycoplasma genitalium & $0 \cdot 566$ & 523 & 179 & 81 & $95 / 53$ & 27 & 4 & $55 / 19$ & 4 & 3 \\
\hline Mycoplasma pneumoniae & $0 \cdot 797$ & 729 & 209 & 121 & $117 / 67$ & 22 & 3 & $81 / 32$ & 7 & 1 \\
\hline Buchnera aphidicola & 0.625 & 596 & 49 & 40 & $24 / 20$ & 5 & 0 & $19 / 18$ & 3 & 0 \\
\hline Clostridium perfringens & $2 \cdot 960$ & 2855 & 174 & 89 & $78 / 90$ & 5 & 1 & $46 / 42$ & 0 & 1 \\
\hline
\end{tabular}




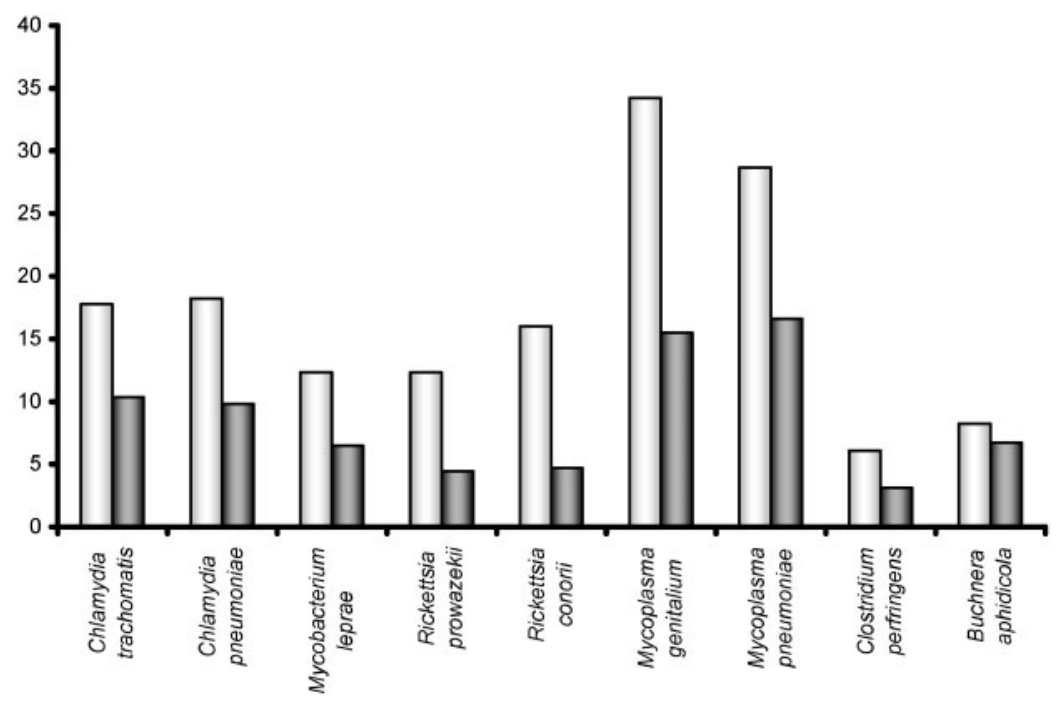

Fig. 1. The percentage of genes that overlap in genomes of nine bacterial species with different lifestyles. Light grey, all genes; dark grey, 'genuine' genes.

Obligatory intracellular parasites follow this trend, thereby endorsing the fact that overlapping genes are a means of compressing the maximum amount of information into a short sequence. Mycobacterium leprae is an exceptional facultative intracellular parasite that has a higher proportion of overlapping genes compared to the free-living anaerobe Clostridium perfringens. This may be explained by the notion that Mycobacterium leprae is still undergoing downsizing and genome reduction, as it is often considered a genome in decay'. This explanation is supported by the fact that Mycobacterium leprae has the maximum number of pseudogenes $(>1000)$ compared to only 12 pseudogenes in $R$. prowazekii, the obligatory intracellular parasite with the most extensive genome degradation (Fig. 1). These observations clearly implicate the role of overlapping genes and their contribution to genome reduction.

When we correlated the genome sizes of the nine bacterial species with the numbers of overlapping genes, a weak correlation of 0.56 was found. Inclusion of Escherichia coli in our data raised the correlation to $0 \cdot 86$. Fukuda et al. (2003) reported a complete analysis of overlapping genes and genome sizes of 50 prokaryotes, and found a good correlation between the number of overlapping genes and genome size. Thus, the niche and lifestyle of the organism also play an important role in determining the proportion of genes showing overlapping configuration, and may be considered as constraints that favour smaller genomes. In particular, these adaptive pressures explain how a given quantity of information can be represented by a relatively small message.

Overlapping gene pairs can assume one of three structures, namely, 'convergent' $(\rightarrow \leftarrow)$, 'unidirectional' $(\rightarrow \rightarrow)$ or 'divergent' $(\leftarrow \rightarrow)$ (Rogozin et al., 2002). A significant proportion $(>90 \%)$ of overlapping gene pairs in all the genomes were identified to be unidirectional. These results support the earlier hypothesis by Eyre-Walker (1995) that most overlapping gene pairs have unidirectional structure (Table 1). The frequent occurrence of the unidirectional overlapping structure probably reflects the commonest orientation of adjacent genes in the chromosomes, as prokaryotic genes are often organized into operons or clusters of genes that are transcribed together. Since all genes in an operon must be transcribed in the same direction, this organization will be reflected by a tendency for adjacent genes to have the same orientation. Fewer gene pairs have the two inverted orientations $(\leftarrow \rightarrow$ and $\rightarrow \leftarrow)$. The lower proportion of the divergent structure may be attributed to the evolutionary constraints at the $5^{\prime}$ end of the gene and the upstream region, which incorporate essential structures such as promoters. In addition, a frameshift mutation at the $5^{\prime}$ end may destroy the entire gene. The unidirectional and convergent structures are more easily formed due to the loss of stop codons or a frameshift. These results concur with those of Rogozin et al. (2002) and Fukuda et al. (2003), and highlight the fact that gene orientation, genome reduction and evolutionary constraints work together during the organism's adaptation in its niche.

\section{Comparative study of overlapping genes in R. prowazekii and $R$. conorii}

Gene pairs that occur as overlapping genes in both genomes. $R$. prowazekii is thought to have essentially appeared as a subset of $R$. conorii 40 to 80 million years ago. One hundred and thirty seven genes of $R$. conorii do not have any sequence similarity with the $R$. prowazekii genome (Ogata et al., 2001). Supplementary Table A (in IJSEM Online) summarizes the overlapping gene pairs in two genomes $-R$. prowazekii and $R$. conorii. The overlapping gene pairs are unidirectional, and are found on the same strand in both the genomes except for RP884/ RP885 $(\leftarrow \leftarrow)$ and RC1373/RC1374 $(\rightarrow \rightarrow)$. The number of unidirectional overlapping gene pairs is more on the leading strand than on the lagging strand for both the 
genomes (Supplementary Table A). This may be attributed to the existence of a larger number of genes in the leading strand (Rocha \& Danchin, 2001). Out of the unidirectional overlaps, many (11/23) are $4 \mathrm{bp}$ in length. Most of the $4 \mathrm{bp}$ overlaps are ATGA, involving fusion of the termination codon of one gene with the initiation codon of another (translational coupling). This observation reveals the role of gene overlaps in bringing neighbouring genes in contact with the translational machinery to ensure some form of coordinated regulation. A similar case has been reported for the trp operon where TrpC and TrpF overlap by several base pairs (Zheng et al., 2002). Six gene pairs were found to be overlapping in both the genomes, with different numbers of bases in overlap. The entries and the causes of change in overlapping nucleotides are shown in Supplementary Table B (in IJSEM Online).

To overlap or not to overlap. Although there are six gene pairs that are overlapping in $R$. prowazekii, these are split in $R$. conorii. However, there are only three gene pairs that are overlapping in $R$. conorii, but are split in $R$. prowazekii, which has a smaller genome than $R$. conorii (Table 1). These differences support the notion that overlapping genes may be a means of compressing the maximum amount of information into the available short sequence space, and may be a result of evolutionary pressure to minimize genome size. It was observed that overlapping genes are generated due to loss of a stop codon or start codon of either gene that results in extension of the $3^{\prime}$ end or reassignment of the start codon. This can happen as a result of deletion of the stop/start codons, point mutations at the stop/start codons or frameshift anywhere in the coding region. The results are elaborated below.

Gene pairs that overlap in $R$. prowazekii but are split in $\boldsymbol{R}$. conorii. Six gene pairs overlap in $R$. prowazekii, but are non-overlapping or split in $R$. conorii. Out of these, two are present with zero intergenic distance between them in $R$. conorii, and have 4 bp overlap in $R$. prowazekii (Supplementary Table C, in IJSEM Online). However, four of them are overlapping in $R$. prowazekii, and have intergenic distances ranging from 3 to $188 \mathrm{bp}$ in R. conorii (Supplementary Table D, in IJSEM Online).

Gene pairs that overlap in $R$. conorii but are split in $\boldsymbol{R}$. prowazekii. Three gene pairs were identified as overlapping in $R$. conorii, but as split genes in $R$. prowazekii. The intergenic distance for these genes ranges from 1 to $40 \mathrm{bp}$. The possible causes for their emergence are elaborated in Supplementary Table E (in IJSEM Online).

\section{Conclusion}

Whole genome sequencing of micro-organisms is providing an opportunity for computer-based genetic analysis that allows us to highlight important features such as overlapping genes in the genomes. From our analysis, mutations at the ends of the coding region are the main force that determines gene overlaps. It also appears that gene overlaps arise from the reduction or elimination of intergenic regions caused by mutational bias towards deletion that helps in genome compression while retaining information content. Furthermore, most of the overlapping genes are not mutually exclusive in function. These studies thus emphasize that there is substantial plasticity among obligatory intracellular parasites, and that overlapping genes facilitate genome reduction and functional coupling.

\section{REFERENCES}

Andersson, J. O. \& Andersson, S. G. (1999). Insights into the evolutionary process of genome degradation. Curr Opin Genet Dev $\mathbf{9}$, 664-671.

Andersson, S. G., Zomorodipour, A., Andersson, J. O. \& 7 other authors (1998). The genome sequence of Rickettsia prowazekii and the origin of mitochondria. Nature 396, 133-140.

Burge, C. B. \& Karlin, S. (1998). Finding the genes in genomic DNA. Curr Opin Struct Biol 8, 346-354.

Cebrat, S., Dudek, M. R., Mackiewicz, P., Kowalczuk, M. \& Fita, M. (1997). Asymmetry of coding versus noncoding strand in coding sequences of different genomes. Microb Comp Genomics 2, 259-268.

Chen, S. M., Takiff, H. E., Barber, A. M., Dubois, G. C., Bardwell, J. C. \& Court, D. L. (1990). Expression and characterization of RNase III and Era proteins. Products of the rnc operon of Escherichia coli. J Biol Chem 265, 2888-2895.

Clark, M. A., Baumann, L., Thao, M. L., Moran, N. A. \& Baumann, P. (2001). Degenerative minimalism in the genome of a psyllid endosymbiont. J Bacteriol 183, 1853-1861.

Cole, S. T., Eiglmeier, K., Parkhill, J. \& 41 other authors (2001). Massive gene decay in the leprosy bacillus. Nature 409, 1007-1011.

Eyre-Walker, A. (1995). The distance between Escherichia coli genes is related to gene expression levels. J Bacteriol 177, 5368-5369.

Fraser, C. M., Gocayne, J. D., White, O. \& 25 other authors (1995). The minimal gene complement of Mycoplasma genitalium. Science 270, 397-403.

Fukuda, Y., Washio, T. \& Tomita, M. (1999). Comparative study of overlapping genes in the genomes of Mycoplasma genitalium and Mycoplasma pneumoniae. Nucleic Acids Res 27, 1847-1853.

Fukuda, Y., Nakayama, Y. \& Tomita, M. (2003). On dynamics of overlapping genes in bacterial genomes. Gene 323, 181-187.

Himmelreich, R., Hilbert, H., Plagens, H., Pirkl, E., Li, B. C. \& Herrmann, R. (1996). Complete sequence analysis of the genome of the bacterium Mycoplasma pneumoniae. Nucleic Acids Res 24, $4420-4449$.

Inokuchi, Y., Hirashima, A., Sekine, Y., Janosi, L. \& Kaji, A. (2000). Role of ribosome recycling factor (RRF) in translational coupling. EMBO J 19, 3788-3798.

Kalman, S., Mitchell, W., Marathe, R. \& 7 other authors (1999). Comparative genomes of Chlamydia pneumoniae and C. trachomatis. Nat Genet 21, 385-389.

Keese, P. K. \& Gibbs, A. (1992). Origins of genes: "big bang” or continuous creation? Proc Natl Acad Sci U S A 89, 9489-9493.

Krakauer, D. C. (2000). Stability and evolution of overlapping genes. Evolution Int J Org Evolution 54, 731-739. 
Lipman, D. J. (1997). Making (anti)sense of non-coding sequence conservation. Nucleic Acids Res 25, 3580-3583.

Miyata, T. \& Yasunaga, T. (1978). Evolution of overlapping genes. Nature 272, 532-535.

Normark, S., Bergstrom, S., Edlund, T., Grundstrom, T., Jaurin, B., Lindberg, F. P. \& Olsson, O. (1983). Overlapping genes. Annu Rev Genet 17, 499-525.

Ogata, H., Audic, S., Renesto-Audiffren, P. \& 8 other authors (2001). Mechanisms of evolution in Rickettsia conorii and $R$. prowazekii. Science 293, 2093-2098.

Pavesi, A., De laco, B., Granero, M. I. \& Porati, A. (1997). On the informational content of overlapping genes in prokaryotic and eukaryotic viruses. J Mol Evol 44, 625-631.

Rocha, E. P. \& Danchin, A. (2001). Ongoing evolution of strand composition in bacterial genomes. Mol Biol Evol 18, 1789-1799.

Rogozin, I. B., Spiridonov, A. N., Sorokin, A. V., Wolf, Y. I., Jordan, I. K., Tatusov, R. L. \& Koonin, E. V. (2002). Purifying and directional selection in overlapping prokaryotic genes. Trends Genet 18, 228-232.
Sander, C. \& Schulz, G. E. (1979). Degeneracy of the information contained in amino acid sequences: evidence from overlaid genes. J Mol Evol 13, 245-252.

Shigenobu, S., Watanabe, H., Hattori, M., Sakaki, Y. \& Ishikawa, H. (2000). Genome sequence of the endocellular bacterial symbiont of aphids Buchnera sp. APS. Nature 407, 81-86.

Shimizu, T., Ohtani, K., Hirakawa, H. \& 7 other authors (2002). Complete genome sequence of Clostridium perfringens, an anaerobic flesh-eater. Proc Natl Acad Sci U S A 99, 996-1001.

Smith, T. F. \& Waterman, M. S. (1981). Overlapping genes and information theory. J Theor Biol 91, 379-380.

Stephens, R. S., Kalman, S., Lammel, C. J. \& 9 other authors (1998). Genome sequence of an obligate intracellular pathogen of humans: Chlamydia trachomatis. Science 282, 754-759.

Yelin, R., Dahary, D., Sorek, R. \& 13 other authors (2003). Widespread occurrence of antisense transcription in the human genome. Nat Biotechnol 21, 379-386.

Zheng, Y., Szustakowski, J. D., Fortnow, L., Roberts, R. J. \& Kasif, S. (2002). Computational identification of operons in microbial genomes. Genome Res 12, 1221-1230. 